
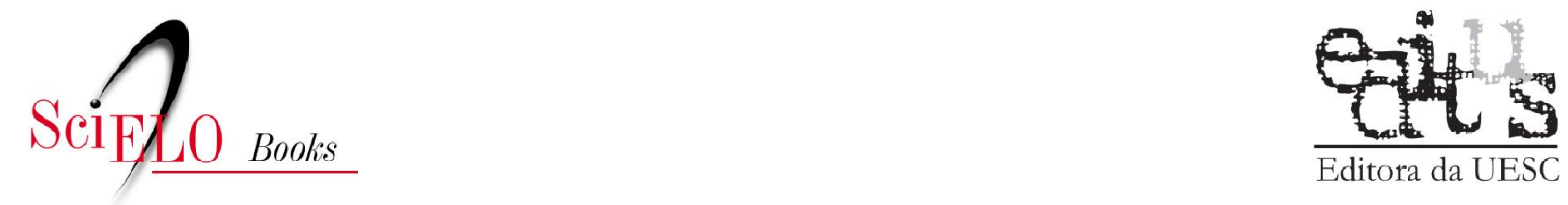

Editora da UESC

\title{
28 - Poneromorfas como indicadoras de impacto pela mineração e de reabilitação após mineração
}

\author{
Ananza Mara Rabello \\ Antônio César Medeiros de Queiroz \\ Carla Rodrigues Ribas
}

\section{SciELO Books / SciELO Livros / SciELO Libros}

RABELLO, AM., QUEIROZ, ACM., and RIBAS, CR. Poneromorfas como indicadoras de impacto pela mineração e de reabilitação após mineração. In: DELABIE, JHC., et al., orgs. As formigas poneromorfas do Brasil [online]. Ilhéus, BA: Editus, 2015, pp. 425-436. ISBN 978-85-7455-441-9.

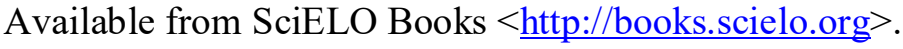

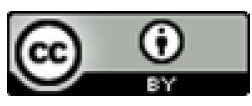

All the contents of this work, except where otherwise noted, is licensed under a Creative Commons Attribution 4.0 International license.

Todo o conteúdo deste trabalho, exceto quando houver ressalva, é publicado sob a licença Creative Commons Atribição 4.0.

Todo el contenido de esta obra, excepto donde se indique lo contrario, está bajo licencia de la licencia Creative Commons Reconocimento 4.0. 


\title{
Poneromorfas como indicadoras de impacto pela mineração e de reabilitação após mineração
}

\author{
Ananza Mara Rabello, Antônio César Medeiros de Queiroz, Carla Rodrigues Ribas
}

\section{Resumo}

O impacto ambiental gerado por atividades antrópicas, como a mineração, leva à perda da biodiversidade local, desestruturação das comunidades biológicas e alterações na estrutura e processos do solo, reduzindo a integridade dos ecossistemas. Uma maneira eficiente de medir e monitorar mudanças ambientais é através de bioindicadores como as formigas, as quais têm sido amplamente utilizadas como bioindicadoras de impacto e de reabilitação. Dentre as formigas, as poneromorfas têm se destacado no Brasil como um agrupamento frequentemente documentado como bioindicador de diversos tipos de impactos antrópicos. Diante disso, neste capítulo, analisamos o uso de poneromorfas como indicadoras de impacto pela mineração e de reabilitação após mineração, além de verificar o papel de poneromorfas na função ecológica de remoção de sementes destas áreas. Nós coletamos 16 espécies de poneromorfas epigeicas, entre essas, cinco foram removedoras de sementes. Nenhuma dessas espécies foi coletada nas áreas em mineração. Nós encontramos uma maior riqueza de poneromorfas epigeicas e removedoras de sementes nas áreas não perturbadas do que nas áreas em reabilitação. Essa diferença entre as áreas não perturbadas e agudas em reabilitação também foi detectada na composição de espécies epigeicas. Assim, as formigas poneromorfas epigeicas e removedoras de sementes são altamente afetadas pelo impacto causado pela mineração, comprometendo o equilíbrio ecológico e o bom funcionamento desses ambientes, uma vez que as mesmas ocupam diferentes níveis tróficos que vão desde oportunistas e generalistas a especialistas. Além disso, as diferentes técnicas de reabilitação não foram suficientes para recuperar a riqueza e a composição originais de poneromorfas, mostrando que a reabilitação com espécies exóticas de capim cria um ambiente diferente daquele com vegetação nativa. As formigas poneromorfas epigeicas e removedoras de sementes se mostram como uma importante ferramenta na bioindicação.

RABELLO, Ananza Mara; QUEIROZ, Antônio César Medeiros de; RIBAS, Carla Rodrigues. Poneromorfas como indicadoras de impacto pela mineração e de reabilitação após mineração. In: DELABIE, Jacques H. C. et al. As formigas poneromorfas do Brasil. Ilhéus: Editus, 2015. p. 425-436. 
Poneromorphs ants as bioindicators of mining and rehabilitation after mining Environmental impacts caused by anthropic activities, such as mining, can lead to biodiversity loss, disruption of biological communities, alterations in soil structure and reductions in the ecosystem 's integrity. An efficient way to measure and monitor environmental change is through bioindicators such as ants, which have been widely used as bioindicators of mining impacts and rehabilitation. Among ants, the poneromorphs have been highlighted in Brazil as a group that is frequently considered as a bioindicator of several anthropic impacts. In this chapter, we analyse the use of poneromorphs as bioindicators of mining impact and minesite rehabilitation, and verify the role of poneromorphs in the ecological function of seed removal in these areas. We collected 16 epigaeic poneromorph species and, among them, five species were seed-removing types. None of these species were collected in mining areas. We found higher epigaeic and seed-removing poneromorph richness in undisturbed areas than in rehabilitation ones. We also detected such differences between undisturbed and rehabilitated areas in the composition of epigaeic species. Thus, the epigaeic and seed-removing poneromorph ants are highly affected by mining impact, and, since they occupy different trophic levels that range from opportunist and generalist to specialist, contribute to the ecological balance and good functioning of these environments. Furthermore, the various rehabilitation techniques that we investigated were not sufficient for recovering the original poneromorph richness and composition, which indicates that rehabilitation with exotic grasses creates an environment different from the original, native one. It is concluded that epigaeic and seedremoving poneromorph ants are an important tool in bioindication.

\section{Introdução}

Atualmente as paisagens tropicais são caracterizadas por mosaicos de habitats naturais como consequência das atividades antrópicas, com intensa exploração dos recursos naturais que causam mudanças drásticas na paisagem (PERFECTO; VANDERMEER, 2008). O impacto ambiental gerado por tais atividades leva à perda $\mathrm{da}$ biodiversidade local, desestruturação das comunidades biológicas e alterações na estrutura e processos do solo, reduzindo a integridade dos ecossistemas (MADEIRA, 2009; GARDNER 2010). A magnitude das ameaças aos ambientes naturais tem chamado a atenção da comunidade científica ao desafio de desenvolver habilidades para medir e monitorar o nível desses impactos ambientais (WILSON, 2000; ALONSO, 2010).

A medida e o monitoramento de mudanças ambientais, os quais são difíceis de avaliar diretamente, podem ser feitos com o uso de bioindicadores (MCGEOCH, 1998). Esses organismos são capazes de fornecer um diagnóstico rápido e eficaz sobre as condições do ambiente, com a vantagem de apresentarem baixo custo financeiro para essa tarefa
(NIEMI; MC DONALD, 2004; GARDNER, 2010).

Sendo assim, os bioindicadores são capazes de fornecer informações objetivas e significativamente importantes para estratégias de conservação.

Invertebrados são amplamente utilizados como bioindicadores por possuírem alta abundância e diversidade, importância ecológica, sensibilidade a variações ambientais e facilidade de amostragem (MCGEOCH, 1998; ANDERSEN et al., 2004; GARDNER, 2010). Somada a tais características, as formigas ainda apresentam ampla distribuição geográfica e são taxonômica e ecologicamente bem conhecidas (PHILPOTT et al., 2010; RIBAS et al., 2012a). Além disso, as formigas desempenham importantes papéis ecológicos em todos os ecossistemas terrestres, tais como: ciclagem de nutrientes (SOUSA-SOUTO et al., 2007), aeração do solo (GABET et al., 2003), predação (PHILPOTT; ARMBRECHT, 2006), controle biológico (SILVA; PERFECTO, 2013) e dispersão de sementes (LEAL et al., 2014).

Por essas razões, as formigas são um grupo proeminentemente usado como uma poderosa ferramenta em estudos sobre impactos ambientais (HOFFMAN, 2010; RIBAS et al., 2012a). Esses 
estudos incluem distúrbios por fogo (PARR et al., 2007), agricultura (DOSSANTOS; PERFECTO, 2011), fragmentação (PAOLUCCI et al., 2012), pastagem (VASCONCELOS, 1999), mineração (HOLEC et al., 2006), recuperação após mineração (MAJER, 2007), dentre outros (RIBAS et al., 2012a).

Dentre essas atividades antrópicas, provavelmente a mineração é uma das mais impactantes, pois muda completamente a paisagem resultando em uma drástica alteração do solo e da biota associada (HOLEC; FROUZ, 2005). Hoje, o Brasil é um dos maiores produtores e exportadores de minério de ferro do mundo, e a Companhia Vale do Rio Doce é a maior responsável por essa produção e uma das maiores empresas de mineração mundial (IBRAM, 2011). Com isso, a atividade de mineração tem significativa contribuição na economia brasileira criando um cenário de dicotomia entre o desenvolvimento econômico e a conservação da biodiversidade.

Com o crescimento da mineração no Brasil tornam-se cada vez mais necessários estudos que abordem a manutenção e a recuperação da biodiversidade e o funcionamento de ecossistemas ameaçados por essa atividade. Tais estudos devem conter uma perspectiva de conservação (biodiversidade) e sustentabilidade (funções ecológicas) para mensurar as possíveis perdas de espécies e também a eficácia das reparações realizadas pela empresa.

No Brasil, os trabalhos que utilizaram formigas como bioindicadoras em áreas de mineração buscaram compreender os efeitos do impacto e como ocorre o processo de recuperação da comunidade de formigas em áreas de reabilitação (e.g. MAJER, 1992; MAJER, 1996; DIEHL et al., 2004; PEREIRA et al., 2007; COSTA et al., 2010; PAIS; VARANDA, 2010; RIBAS et al., 2012b), além da resposta à presença de metais pesados (RIBAS et al., 2012c). Tais trabalhos mostraram um efeito negativo na riqueza de espécies de formigas e mudanças na composição frente ao impacto, bem como uma relação positiva entre o tempo de reabilitação e a riqueza de formigas (veja COSTA et al., 2010; PAIS; VARANDA, 2010; RIBAS et al., 2012b). Esses trabalhos realizados no Brasil não avaliaram o impacto da mineração e os esforços de reabilitação pós-mineração sobre o funcionamento do ecossistema, através de funções ecológicas desempenhadas pelas formigas, como por exemplo, a dispersão de sementes (mirmecocoria).
A Mirmecocoria pode ser negativamente afetada pela mineração que provoca mudanças na riqueza e composição de espécies de formigas removedoras de sementes (DOMINGUEZ-HAYDAR; ARMBRECHT, 2011; HENAO-GALLEGO et al., 2012). Isso é preocupante já que as formigas trazem benefícios para as plantas por evitar que as sementes sejam predadas por granívoros (ANDERSEN; MORRISON, 1998). Grant et al. (2007) mostraram que a presença de sementes no solo é essencial para a reabilitação de áreas pós-distúrbio. Assim, tais formigas podem funcionar como importantes agentes no processo de reabilitação tornando-o autossustentável por agir na distribuição e favorecer a germinação de novos indivíduos de espécies de plantas (LOMOV et al., 2009).

Dentre as formigas, as poneromorfas têm se destacado no Brasil como um importante agrupamento removedor de sementes, por possuírem corpo grande e assim carregarem as sementes em distâncias maiores (ALMEIDA et al., 2013; LEAL et al., 2014). Além disso, as poneromorfas têm sido documentadas em outras associações mutualísticas com as plantas, como com nectários extraflorais (PEREIRA et al., 2013). Porém, sua importância ecológica vai além de interações com as plantas, pois as poneromorfas ocupam diferentes níveis tróficos nos ecossistemas, como predadoras generalistas e onívoras (COGNI; OLIVEIRA, 2004; BRANDÃO et al., 2011).

Outro destaque é para o fato de que as poneromorfas frequentemente têm sido documentadas como bioindicadoras de diversos tipos de impactos antrópicos (RIBAS et al., 2012a). No Brasil, impactos causados por pastagem (Anochetus e Odontomachus), Eucalyptus (Ectatomma, Hypoponera, Odontomachus e Pachycondyla) e fogo (Ectatomma e Odontomachus), bem como áreas não perturbadas (Anochetus, Gnamptogenys, Odontomachus e Pachycondyla) apresentam formigas poneromorfas como bioindicadoras (MARINHO et al., 2002; RAMOS et al., 2003; DIAS et al., 2008; ENDRINGER et al., 2008; SCHMIDT; DIEHL, 2008; ILHA et al., 2009; BRAGA et al., 2010). No contexto da mineração e reabilitação de áreas pós-mineração, no Brasil, os gêneros Ectatomma, Hypoponera e Pachycondyla aparecem como bioindicadores (DIEHL et al., 2004; PEREIRA et al., 2007; RIBAS et al., 2012 b,c).

Assim, neste trabalho nós avaliamos o uso de poneromorfas como indicadoras de impacto pela mineração e de reabilitação após mineração, 
além de verificar o papel de poneromorfas na função ecológica de remoção de sementes destas áreas. Para isso, nós avaliamos os seguintes parâmetros: (1) riqueza e composição de formigas poneromorfas; (2) riqueza e composição de poneromorfas removedoras de sementes; (3) poneromorfas exclusivas de impacto pela mineração e de diferentes tempos de reabilitação após mineração.

\section{Material e Métodos}

\section{Área de estudo}

Nós realizamos as coletas nos meses de fevereiro e março de 2012 em três unidades da empresa Vale S.A.: Mina do Tamanduá $\left(20^{\circ} 5^{\prime} 17^{\prime \prime}\right.$ S e $\left.43^{\circ} 56^{\prime} 27^{\prime \prime} \mathrm{W}\right)$, Mina Capão Xavier $\left(20^{\circ} 2^{\prime} 47^{\prime \prime} \mathrm{S}\right.$ e $43^{\circ} 58^{\prime} 59^{\prime \prime}$ W) e Mina da Mutuca $\left(20^{\circ} 1^{\prime} 43^{\prime \prime}\right.$ S e $43^{\circ} 57^{\prime} 10^{\prime \prime}$ W) localizadas em Nova Lima, Região Metropolitana de Belo Horizonte, Minas Gerais, Sudeste do Brasil. A altitude média dessas áreas é de $1400 \mathrm{~m}$. O clima local apresenta invernos secos, de abril a setembro, e verões chuvosos, de outubro a março.

Nas três unidades amostramos áreas não perturbadas (controle), em reabilitação no entorno da cava ou sobre pilha de estéril e com atividade de mineração (perturbadas). Tais áreas apresentam a ocorrência de campos ferruginosos compostos por formações abertas, como campos sujos, campos rupestres sobre canga e formações florestais, e estão inseridos na transição entre os biomas Cerrado e Mata Atlântica (RIZZINI, 1997).

Amostramos quatro áreas não perturbadas em formações abertas (campo sujo) no entorno da cava, duas áreas em reabilitação sobre a rocha da cava pós-lavra, reabilitadas por capim gordura (Melinis minutiflora P. Beauv.) e seis áreas com atividade de mineração nas unidades da Mina do Tamanduá e Mina Capão Xavier. Na Mina da Mutuca amostramos duas áreas não perturbadas em matas ciliares e cinco áreas em reabilitação sobre pilha de estéril com diferentes idades e tipos de reabilitação. Duas destas áreas de reabilitação são mais recentes, com dois e quatro anos, e são dominadas por capim gordura e feijão guandu (Cajanus cajan (L.) Millsp). As outras três áreas possuíam seis, oito e dez anos de reabilitação e eram dominadas por capim braquiária (Brachiaria decumbens Stapf), além de feijão guandu e outras plantas nativas que colonizaram o local naturalmente. Assim, no total, nós amostramos seis áreas não perturbadas, sete em reabilitação e seis com atividade de mineração.

\section{Amostragem}

Nós demarcamos 19 transectos, um em cada uma das áreas descritas anteriormente. Em cada transecto amostramos dez pontos com distância mínima de $20 \mathrm{~m}$ entre eles para avaliar as poneromorfas epigeicas. Em cada ponto instalamos armadilhas do tipo pitfall epigeico sem iscas a fim de amostrar este segmento da comunidade de formigas (BESTELMEYER et al., 2000). As 190 armadilhas permaneceram em campo por 48 horas.

Para a amostragem das formigas removedoras de sementes, em cinco pontos por transecto, 95 pontos no total, nós ofertamos 50 frutos artificiais ricos em lipídios (veja RAIMUNDO et al., 2004) protegidos da predação por vertebrados por uma gaiola metálica instalada com a altura de $1,5 \mathrm{~cm}$. Os frutos artificiais continham uma parte fresca que representou o elaiossomo. Esta parte consistiu de gordura vegetal $(75 \%)$, frutose $(4,8 \%)$, sacarose $(0,5 \%)$, glicose $(4,7 \%)$, caseína (7\%), carbonato de cálcio (3\%) e maltodextrina (5\%), e foi desenvolvida no Laboratório de Química, Bioquímica e Análises de Alimentos (Departamento de Engenharia de Alimentos, Universidade Federal de Lavras, Brasil). Para representar as sementes nós utilizamos miçangas laranja de $0,03 \mathrm{~g}$ e $2 \mathrm{~mm}$ de diâmetro. O fruto (miçanga + parte fresca) possuía um peso total de $0,2 \mathrm{~g}$ e 5,5 $\mathrm{mm}$ de diâmetro. Estas características os classificam como frutos de tamanho pequeno e médio (PIZO; OLIVEIRA, 2001). Nós ofertamos os frutos às 09:00h e checamos cada ponto em períodos de 15 minutos até as 16:00h. Em cada observação, nós coletamos as espécies de formigas atraídas que removiam os frutos a uma distância maior que $30 \mathrm{~cm}$ do local de oferta (CHRISTIANINI; OLIVEIRA, 2010).

Após as coletas nós fizemos a identificação dos gêneros de formigas com o auxílio de chaves de identificação (BOLTON, 1994; PALACIO; FERNÁNDEZ, 2003) e as morfoespeciamos para calcular a riqueza e composição de espécies presentes em cada armadilha. Essa identificação foi checada pelo especialista Rodrigo Feitosa, da Universidade Federal do Paraná. Exemplares de todas as morfoespécies foram depositados na coleção de referência do Laboratório de Ecologia de Formigas da Universidade Federal de Lavras - UFLA. 


\section{Análise dos dados}

Para identificar os efeitos do impacto da mineração na riqueza de poneromorfas epigeicas e removedoras nós construímos modelos lineares generalizados (GLM). Nós realizamos análises com a riqueza total de espécies epigeicas e com as removedoras separadamente. Nos modelos, a riqueza de formigas foi utilizada como variável resposta (y) e as áreas (não perturbadas, em reabilitação e em mineração) foram utilizadas como variáveis explicativas $(\mathrm{x})$. Todas as variáveis foram analisadas na escala transecto $(\mathrm{N}=19)$. Os modelos foram construídos com distribuição de erros Poisson adequados para dados de contagem. As análises de resíduos apontaram a distribuição de erros utilizada como a mais adequada.

Para verificarmos os padrões da composição de espécies de poneromorfas epigeicas nas áreas, nós plotamos um NMDS (non-metric multidimensional scaling) e, posteriormente, realizamos análises de similaridade (ANOSIM) usando o índice de similaridade de Jaccard, adequado para matrizes de presença e ausência. Não foi possível realizarmos essa análise para as formigas poneromorfas removedoras de sementes, pois nas áreas em reabilitação coletamos tais formigas apenas em um ponto amostral.
As análises de dados foram feitas no software R 3.0.1 (R Development Core Team, 2013), sendo que a NMDS e a ANOSIM foram feitas com o auxílio do pacote vegan. Em nenhuma das análises nós utilizamos as áreas em mineração, pois nenhuma poneromorfa foi coletada nessas áreas.

Adicionalmente, nós plotamos um diagrama de Venn para observarmos o número de espécies de poneromorfas epigeicas e removedoras exclusivas e compartilhadas entre as áreas.

\section{Resultados}

Nós encontramos 16 espécies de poneromorfas distribuídas entre sete gêneros (uma Anochetus, duas Ectatomma, duas Odontomachus, três Gnamptogenys, duas Pachycondyla, duas Neoponera e quatro Hypoponera) pertencentes a duas subfamílias (Ectatomminae e Ponerinae). Dentre as espécies coletadas apenas cinco são removedoras de sementes (Tabela 28.I).

As espécies mais comuns das áreas não perturbadas e que foram coletadas exclusivamente nestas áreas são Ectatomma edentatum, Neoponera marginata e Odontomachus chelifer. Por sua vez, Neoponera verenae é comum em áreas de reabilitação e foi coletada exclusivamente nestas áreas. A espécie Gnamptogenys striatula é comum em ambos os ambientes.

TABELA 28.I - Espécies de formigas poneromorfas em áreas não perturbadas e em reabilitação. Todas as espécies foram coletadas em unidades de mineração da companhia Vale S.A., em Nova Lima, MG, Brasil. *Espécies de formigas poneromorfas removedoras de sementes.

\begin{tabular}{c|c|c|c}
\hline Subfamílias & Espécies & $\begin{array}{c}\text { Áreas não } \\
\text { perturbadas }\end{array}$ & $\begin{array}{c}\text { Áreas em } \\
\text { reabilitação }\end{array}$ \\
\hline Ectatomminae & Ectatomma edentatum* (Roger, 1863) & $\mathrm{X}$ & \\
\hline & $\begin{array}{c}\text { Ectatomma lugens } \\
\text { (Emery, 1894) }\end{array}$ & $\mathrm{X}$ & \\
\hline & Gnamptogenys acuminata (Emery, 1896) & $\mathrm{X}$ & $\mathrm{X}$ \\
\hline & Gnamptogenys striatula* (Mayr, 1884) & $\mathrm{X}$ & $\mathrm{X}$ \\
\hline & Gnamptogenys sp.1 & $\mathrm{X}$ & \\
\hline Ponerinae & Anochetus neglectus (Emery, 1894) & $\mathrm{X}$ & \\
\hline & Hypoponera sp.1 & & $\mathrm{X}$ \\
\hline & Hypoponera sp.2 & $\mathrm{X}$ & \\
\hline & Hypoponera sp.3 & $\mathrm{X}$ & $\mathrm{X}$ \\
\hline & Hypoponera sp.4 & $\mathrm{X}$ & \\
\hline & Odontomachus chelifer* (Latreille, 1802) & $\mathrm{X}$ & \\
\hline & Odontomachus meinerti (Forel, 1905) & $\mathrm{X}$ & \\
\hline & Neoponera verenae* (Forel, 1922) & $\mathrm{X}$ & \\
\hline & Neoponera marginata (Roger, 1861) & $\mathrm{X}$ & \\
\hline & Pachycondyla striata* (Smith, 1858) & & \\
\hline & Pachycondyla harpax (Fabricius, 1804) & 16 &
\end{tabular}


Doze espécies, dentre elas três removedoras, são exclusivas das áreas não perturbadas. Quatro espécies, inclusive uma removedora, são exclusivas das áreas em reabilitação. Duas espécies, sendo que uma é removedora, são compartilhadas entre as áreas não perturbadas e as áreas em reabilitação. Nenhuma espécie de poneromorfa foi amostrada em áreas de mineração (Tabela 28.I, Figura 28.1).

FIGURA 28.1 - Diagrama de Venn com o número de espécies de formigas poneromorfas compartilhadas e exclusivas das áreas não perturbadas, em mineração e reabilitação em unidades de mineração da companhia Vale S.A., em Nova Lima, MG, Brasil

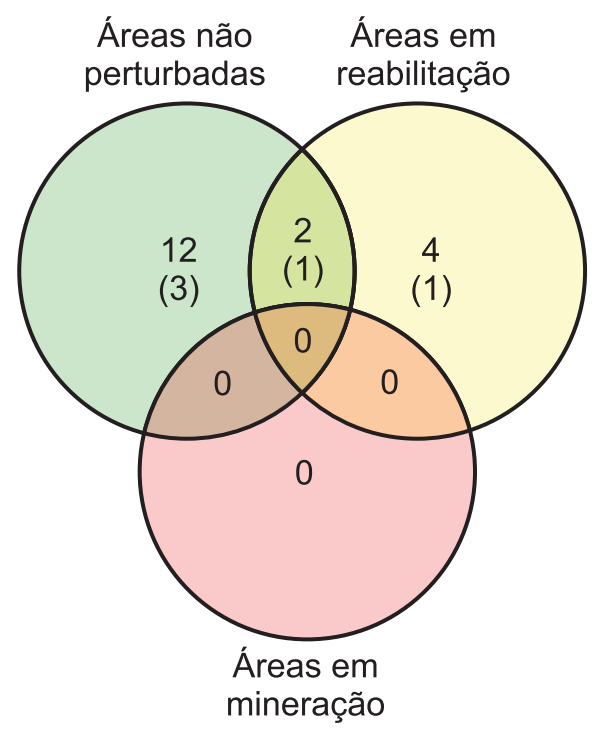

FIGURA 28.2 - Riqueza de espécies de formigas poneromorfas epigeicas em áreas não perturbadas e em reabilitação $(\mathrm{p}=$ 0,0015). Barras representam o erro padrão. As espécies foram coletadas nas unidades de mineração da companhia Vale S.A., em Nova Lima, MG, Brasil

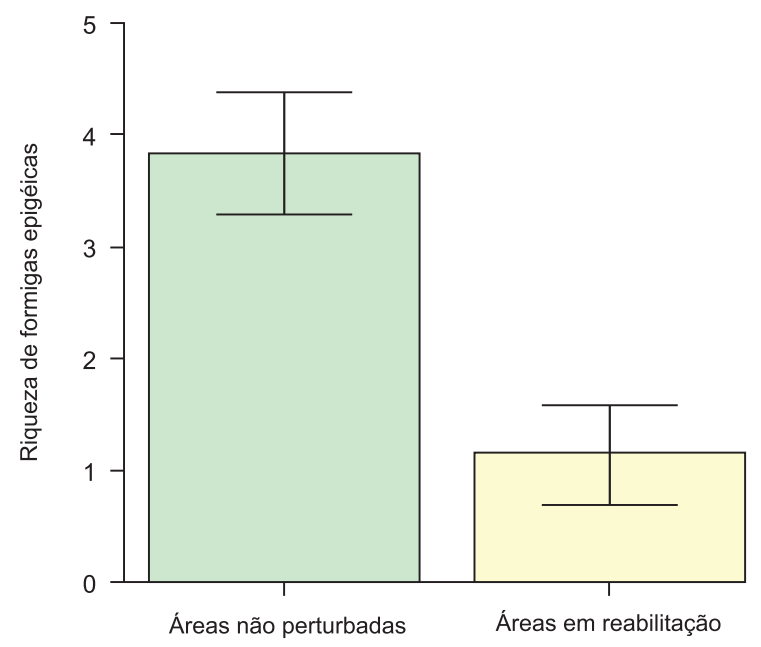

Houve diferença na riqueza de espécies de Poneromorfas epigeicas $\left(\mathrm{p}=0,0015 ; \mathrm{df}=11 ; \chi^{2}=\right.$; Figura $28.2)$ e removedoras $\left(\mathrm{p}=0,0002 ; \mathrm{df}=11 ; \chi^{2}=\right.$; Figura 28.3) entre as áreas, sendo que a riqueza de espécies de ambas foi maior nas áreas não perturbadas do que nas áreas em reabilitação. A composição de espécies epigeicas das áreas não perturbadas é diferente das áreas em reabilitação $(p=0,0070 ; R=0,7163$; Figura 28.4).

FIGURA 28.3 - Riqueza de espécies de formigas poneromorfas removedoras de sementes em áreas não perturbadas e em reabilitação $(\mathrm{p}=0,0002)$. Barras representam o erro padrão. As espécies foram coletadas nas unidades de mineração da companhia Vale S.A., em Nova Lima, MG, Brasil

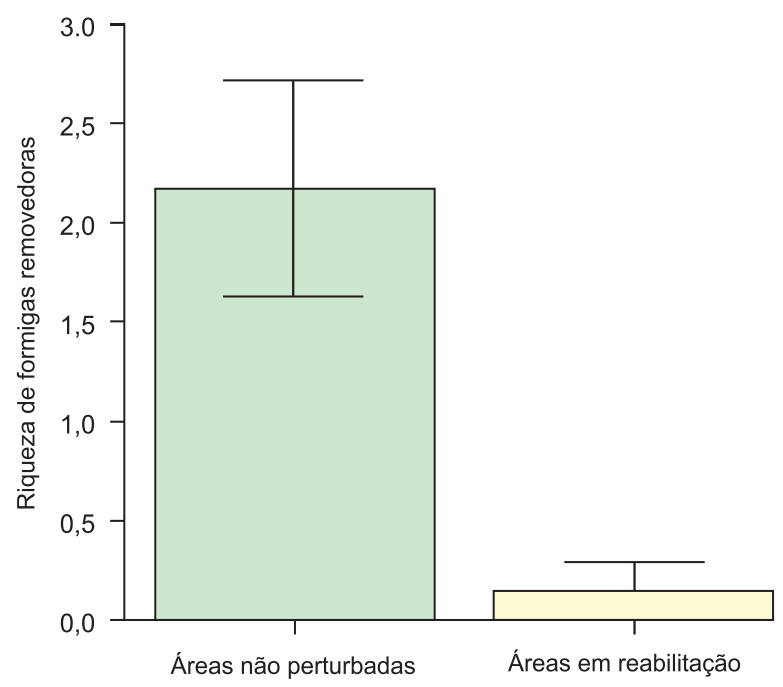

FIGURA 28.4 - Non-metric multidimensional scaling (NMDS), com o uso do índice de Jaccard, utilizando a presença/ausência de espécies de formigas poneromorfas epigeicas em áreas não perturbadas e em reabilitação. Todas as espécies foram coletadas nas unidades de mineração da companhia Vale S.A., em Nova Lima, MG, Brasil

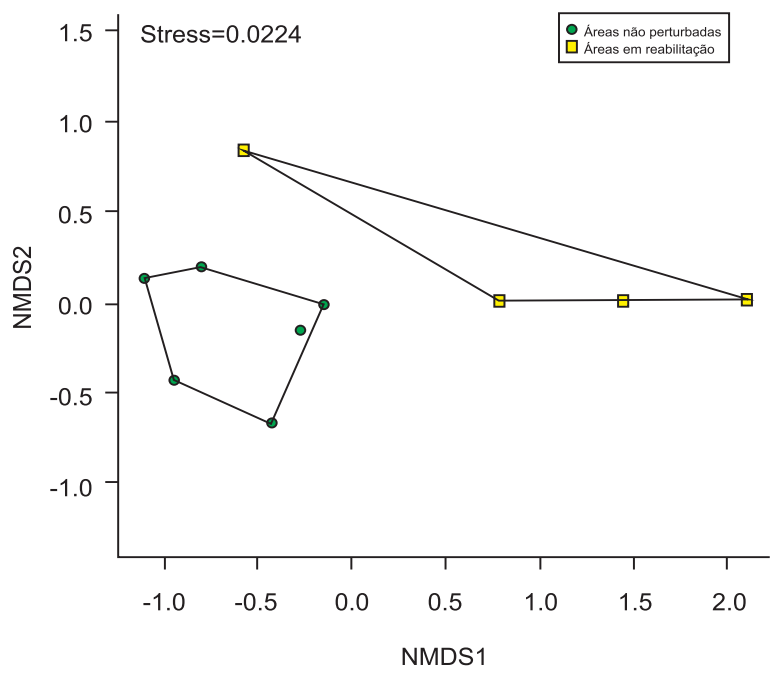


Discussão

As formigas poneromorfas epigeicas e removedoras de sementes são altamente afetadas, nos parâmetros de riqueza e composição, pelo impacto causado pela mineração. Isso gera assembleias distintas em cada área, com maior riqueza de espécies nas áreas não perturbadas do que nas áreas em reabilitação e com efeito ainda maior nas áreas mineradas, aonde não foram coletadas espécies de poneromorfas. Isso ocorre provavelmente pelo fato de as áreas não perturbadas apresentarem maior quantidade e qualidade de recursos e melhores condições para a distribuição das formigas.

Tais diferenças ocorreram porque a mineração é a forma mais intensa de impacto antrópico, com a retirada total da vegetação e camadas de solo para a extração do minério (HOLEC; FROUZ, 2005). Assim, nessas áreas ocorre uma drástica mudança no perfil do solo, que se torna mais compactado e sem matéria orgânica, com consequências negativas para as formigas (MAJER et al., 1984, 2007; DIEHL et al., 2004). De acordo com os nossos resultados, essa mudança levou a perda total de poneromorfas nas áreas em mineração, significando também perda total da função ecológica de remoção de sementes desempenhada por essas formigas.

As formigas poneromorfas são altamente associadas ao desenvolvimento de uma estrutura da vegetação adequada, por esta mesma representar diversificação de condições (ex.: luz e umidade) e recursos (ex.: alimento e locais para nidificação) importantes para a manutenção das suas colônias (RIBAS et al., 2003; LEAL et al., 2012). Ao contrário disso, áreas em mineração são desprovidas de vegetação oferecendo condições adversas a essas formigas e totalmente diferentes das áreas não perturbadas.

Já os ambientes não perturbados tendem a apresentar maior riqueza de formigas poneromorfas do que áreas em reabilitação e áreas mineradas devido à estrutura da vegetação que sustentam, refletindo no desenvolvimento de cobertura de dossel, sub-bosque e camada de serapilheira. Essa estrutura da vegetação mais complexa fornece maior quantidade de microhabitats capazes de abrigar um número maior de espécies (RIBAS et al., 2003; CAMPOS et al., 2006; NEVES et al., 2012). Nossos resultados corroboram essas expectativas teóricas, pois a riqueza de espécies de formigas poneromorfas epigeicas e removedoras de sementes foi maior nas áreas não perturbadas do que nas áreas em mineração e em reabilitação.
As variáveis ambientais presentes em áreas não perturbadas citadas anteriormente são consideradas as mais importantes para as formigas poneromorfas porque podem representar habitats mais heterogêneos e melhores condições microclimáticas para elas (CAMPOS et al., 2007; COSTA et al., 2011). A presença de serapilheira, por exemplo, é um fator importante para poneromorfas epigeicas por ser o principal local de forrageamento dessas formigas, capaz de afetar a presença de algumas espécies (VASCONCELOS et al., 2000; QUEIROZ et al., 2013). Além disso, a serapilheira é uma variável crucial para poneromorfas removedoras de sementes, pois representa um estoque de sementes (DIAS et al., 2012).

Podemos também perceber o alto grau do impacto causado pela mineração através da diferença de riqueza de formigas poneromorfas epigeicas entre áreas não perturbadas e em reabilitação, que foi mais de três vezes maior em áreas não perturbadas, mostrando que 10 anos de reabilitação (nossa área com maior idade) e as diferentes técnicas de reabilitação não foram suficientes para recuperar a riqueza de poneromorfas original. A reabilitação com espécies exóticas de capim cria um ambiente diferente daquele com vegetação nativa, além de atrasar o processo de reabilitação e de colonização de plantas e de formigas poneromorfas (WALKER; DEL MORAL, 2003; RIBAS et al., 2012b). Assim, esse tipo de reabilitação gera um ambiente simplificado com baixa quantidade de matéria orgânica, dificultando a recolonização da flora e fauna nativa (RIZALI et al., 2013).

Tal reabilitação também não foi eficiente para permitir a recolonização de poneromorfas removedoras de sementes especialistas de habitats preservados como Odontomachus chelifer e Pachycondyla striata (SCHMIDT et al., 2013), as quais também são consideradas boas removedoras de sementes (CHRISTIANINI; OLIVEIRA, 2010), e que foram coletadas neste trabalho exclusivamente em áreas não perturbadas. Essas formigas são consideradas boas removedoras de sementes por carregarem as sementes individualmente $\mathrm{e}$ por longas distâncias, o que favoreceria o estabelecimento de diferentes espécies de plantas contribuindo para o processo autossustentável de reabilitação. Coletamos apenas duas espécies de poneromorfas removedoras de sementes, Neoponera verenae e Gnamptogenys striatula, em áreas de reabilitação sendo que apenas a segunda espécie foi também coletada em áreas não perturbadas, 
indicando que a função ecológica de remoção de sementes não está sendo recuperada.

Nós também encontramos diferença na composição de poneromorfas epigeicas entre as áreas. Isso mostra que as áreas em reabilitação e não perturbadas sustentam assembleias de poneromorfas epigeicas altamente dissimilares $(R=0,7163) \mathrm{em}$ relação às espécies que as compõem. Isso pode ser embasado nas doze espécies de poneromorfas epigeicas exclusivas de áreas não perturbadas, e nas quatro espécies exclusivas de áreas em reabilitação, com somente duas espécies em comum entre as áreas. Essa diferença na composição pode estar relacionada às diferenças estruturais entre as áreas, com distintos substratos e complexidade da vegetação.

Somado a isso, as áreas não perturbadas apresentam assembleia de poneromorfas epigeicas mais complexa com diferentes níveis tróficos, com a presença de espécies oportunistas e preferencialmente predadoras generalistas de serapilheira como as dos gêneros Anochetus, Odontomachus, Pachycondyla, Neoponera e Gnamptogenys (COGNI; OLIVEIRA, 2004; BRANDÃO et al., 2011). Dentre estas, exceto as pertencentes ao primeiro gênero, são espécies removedoras de sementes. Além disso, tais áreas possuem espécies especialistas de ambientes preservados como as do gênero Odontomachus e Gnamptogenys, bem como espécies onívoras e generalistas como as do gênero Ectatomma (CARVALHO et al., 2004; WEISER; KASPARI, 2006). Essa complexidade de níveis tróficos tem importância fundamental no equilíbrio ecológico do ambiente, pois mantém interações tróficas e mecanismos funcionais para o bom funcionamento do ambiente (CAUT et al., 2014).

Entretanto, as áreas em reabilitação possuem uma assembleia mais simplificada, contendo maior número de espécies generalistas e oportunistas típicas de ambientes ainda perturbados como as do gênero Hypoponera e Pachycondyla (COSTA et al., 2010; LEAL et al., 2012) e menos espécies especialistas pertencentes por exemplo ao gênero Gnamptogenys. Hoffman; Andersen (2003) e Dominguez-Haydar; Armbrecht (2011) relataram essa mesma diferença na complexidade de níveis tróficos entre comunidades de poneromorfas de áreas não perturbadas e áreas em reabilitação pós-mineração. Portanto nossos resultados corroboram evidências já existentes de que o impacto da mineração causa mudanças na composição e estrutura da assembleia de formigas poneromorfas epigeicas, mesmo em longo prazo, uma vez que áreas com 10 anos de reabilitação ainda apresentam uma assembleia de poneromorfas alterada.

A partir dos nossos resultados, é possível observar como a atividade da mineração é capaz de afetar fortemente, e em longo prazo, as formigas poneromorfas epigeicas e removedoras de sementes comprometendo o equilíbrio ecológico e o bom funcionamento dos ambientes sob essa atividade antrópica. A alteração nos parâmetros de riqueza e composição dessas formigas poneromorfas nos mostra, em curto prazo, que essa é uma excelente métrica para entendermos as respostas dos organismos frente à atividade de mineração, e eficaz na elaboração de estratégias de conservação. Além disso, mostra a importância da abordagem da função ecológica de remoção de sementes como importante ferramenta na bioindicação.

Em programas de monitoramento, onde o tempo e os recursos são limitados, focar em um agrupamento pode ajudar a gerar informações rápidas e de qualidade. Nesse caso, a avaliação das formigas poneromorfas epigeicas juntamente com as removedoras de sementes com certeza trará informações confiáveis e eficientes em programas de monitoramento que precisem de respostas rápidas e em pequena escala e, com isso, facilitar a interação entre conservação e desenvolvimento econômico.

\section{Agradecimentos}

Este trabalho é resultado do projeto de pesquisa: FAPEMIG - CRA RDP 00123-10 - "Biodiversidade e funções ecológicas de formigas - bioindicação de impactos ambientais e de recuperação de áreas degradadas". Nós agradecemos à companhia Vale S.A. por permitir a amostragem das formigas nas suas áreas de mineração e reabilitação, ao Ramon Braga e Cássio Mendanha, por nos ajudar na escolha dessas áreas e ao Anderson Matos, por nos conduzir dentro de cada unidade de mineração. Agradecemos também ao Laboratório de Análises Químicas, Bioquímicas e de Alimentos (Departamento de Engenharia de Alimentos da Universidade Federal de Lavras), pelo espaço e material fornecidos para a confecção dos frutos artificiais. Nós somos gratos aos colegas do LEF: C.J. Lasmar e E.O. Canedo-Junior, F.A. Schmidt, F. Tanure, R.G. Cuissi, T. Moretti por nos ajudar no trabalho de campo e no laboratório. Somos gratos também ao R. Feitosa, G. Camacho e a D. L. Braga, por verificarem a identificação das formigas. Os autores receberam apoio financeiro da CAPES e FAPEMIG. 


\section{Referências}

ANDERSEN, A.; FISCHER, A.; HOFFMANN, B. D.; READ, J. L.; RICHARDS, R. Use of terrestrial invertebrates for biodiversity monitoring in Australian rangelands, with particular reference to ants. Austral Ecology, v. 29, n. 1, p. 87-92, 2004.

ANDERSEN, A. N. AND MORRISON, S. C. Myrmecochory in Australia's seasonal tropics : Effects of disturbance on distance dispersal. Australian Journal of Ecology, v. 23, n. 5, p. 483-491, 1998..

ALMEIDA, F. S.; MAYHÉ-NUNES, A. J.; QUEIROZ, J. M. The importance of Poneromorph ants for seed dispersal in altered environments. Sociobiology, v. 60, n. 3, p. 229-235, 2013.

ALONSO, L. E. Ant Conservation: current status and a call to action. In: Lach, L.; Parr, C.L.; Abbott, K. L. (Ed). Ant ecology. Oxford: Oxford University, p. 5974, 2010.

BESTELMEYER, B. T.; AGOSTI, D.; LEEANNE, F.; ALONSO, T.; BRANDÃO, C. R. F.; BROWN, W. L.; DELABIE, J. H. C.; SILVESTRE, R. Field techniques for the study of ground-living ants: An Overview, description, and evaluation. In: Agosti, D.; Majer, J.D.; Tennant, A.; Schultz, T. (eds), Ants: Standart Methods for Measuring and Monitoring Biodiversity. Smithsonian Institution Press, p. 122$144,2000$.

BOLTON, B. Identification Guide to the Ant Genera of the World. Harvard University Press, Cambridge, 1994.

BRAGA, D. L.; LOUZADA, J.N. C.; ZANETTI, R.; DELABIE, J. Rapid evaluation of ant diversity in land use systems in Southern Bahia, Brazil. Neotropical Entomology, v. 39, n. 4, p. 464-469, 2010.

BRANDÃO, C. R.; SILVA, R. R.; DELABIE, J. H. Neotropical ants (Hymenoptera) functional groups: nutritional and applied implications. Insect Bioecology and Nutrition for Integrated Pest Management, p. 213-236, 2011.

CAMPOS, R. I.; SOARES, J. P.; MARTINS, R. P.; RIBEIRO, S. P. Effect of habitat structure on ant assemblages (Hymenoptera: Formicidae) associated to two pioneer tree species. Sociobiology, v. 47, n. 3, p. 721-737, 2006.

CAMPOS, R. B. F.; SCHOEREDER, J. H.; SPERBER, C. F. Small-scale patch dynamics after disturbance in litter ant communities. Basic and Applied Ecology, v. 8, n. 1, p. 36-43, 2007.
CARVALHO, K. S.; SOUZA A. L. B.; PEREIRA M. S.; SAMPAIO C. P.; DELABIE J. H. C. Comunidade de formigas epígeas no ecótono Mata de Cipó, Domínio de Mata Atlântica, BA, Brasil. Acta Biologica Leopoldensia, v. 26, n. 2, p. 249-257, 2004.

CAUT, S.; JOWERS, M. J.; ARNAN, X.; PEARCE-DUVET, J.; RODRIGO, A.; CERDA, X.; BOULAY, R. R. The effects of fire on ant trophic assemblage and sex allocation. Ecology and Evolution, v. 4, n. 1, p. 35-49, 2014.

CHRISTIANINI, A. V. OLIVEIRA, P. S. Birds and ants provide complementary seed dispersal in a neotropical savanna. Journal of Ecology, v. 98, n. 3, p. 573-582, 2010.

COGNI, R.; OLIVEIRA, P. S. Patterns in foraging and nesting ecology in the neotropical ant, Gnamptogenys moelleri (Formicidae, Ponerinae). Insectes Sociaux, v. 51, n. 2, p. 123-130, 2004.

COSTA, C. B.; RIBEIRO, S. P.; CASTRO, P. T. A. Ants as Bioindicators of Natural Succession in Savanna and Riparian Vegetation Impacted by Dredging in the Jequitinhonha River Basin, Brazil. Restoration Ecology, v. 18, n. s1, p. 148-157, 2010.

COSTA, F. V.; NEVES, F. S.; SILVA, J. O.; FAGUNDES, M. Relationship between plant development, tannin concentration and insects associated with Copaifera langsdorffii (Fabaceae). Arthropod Plant Interactions, v. 5, n. $1,2011$.

DIAS, N. S.; ZANETTI, R.; SANTOS, M. S.; LOUZADA, J.; DELABIE, J. Interaction between forest fragments and adjacent coffee and pasture agroecosystems: responses of the ant communities (Hymenoptera, Formicidae). Iheringia - Serie Zoologia, v. 98, n. 1, p. 136-142, 2008.

DIAS, A. T.; BOZELLI, R. L.; DARIGO, R. M.; ESTEVES, F. D. A.; DOS SANTOS, H. F.; FIGUEIREDO-BARROS, M. P.; NUNES, M. F. Q. S.; ROLAND, F.; ZAMITH, L. R.; SCARANO, F. R. Rehabilitation of a Bauxite Tailing Substrate in Central Amazonia: The Effect of Litter and Seed Addition on Flood-Prone Forest Restoration. Restoration Ecology, v. 20, n. 4, p. 483-489, 2012.

DIEHL, E.; SANHUDO, C. E.; DIEHL-FLEIG, E. Ground-dwelling ant fauna of sites with high levels of copper. Brazilian Journal of Biology, v. 64, n. 1, p. 33-39, 2004.

DOMINGUEZ-HAYDAR, Y;; ARMBRECHT,

I. Response of Ants and Their Seed Removal in Rehabilitation Areas and Forests at El Cerrejón Coal Mine in Colombia. Restoration Ecology, v. 19, n. 201, p. 178-184, 2011. 
DOS SANTOS, I. A.; PERFECTO, I. Impacts of agroecosystems on ant biodiversity in the Amazon in Brazil. Forestry Research Newsletter, v. 18, p. 20-29, 2011.

ENDRINGER, F. B.; SANTOS, I. A.; TEIXEIRA, M. C.; SCHOEREDER, J. H. Ant species richness in sand dune environments following burning (Hymenoptera: Formicidae). Sociobiology, v. 51, n. 2, p. 415-423, 2008.

GABET, E. J.; REICHMAN, O. J.; SEABLOOM, E. $\mathrm{W}$. The effects of bioturbation on soil processes and sediment transport. Annual Review of Earth and Planetary Science, v. 31, n. 1, p. 249-273, 2003.

GARDNER, T. A. Monitoring Forest Biodiversity: Improving Conservation Through EcologicallyResponsible Management. London: Earthscan, p. 360, 2010.

GRANT, C. D.; WARD, S. C.; MORLEY, S. C. Return of ecosystem function to restored bauxite mines in Western Australia. Restoration Ecology, v. 15, n. s4, p. S94-S103, 2007.

HENAO-GALLEGO, N.; ESCOBAR-RAMÍREZ, S.; CALLE, Z.; MONTOYA-LERMA, J.; ARMBRECHT, I. An artificial aril designed to induce seed hauling by ants for ecological rehabilitation purposes. Restoration Ecology, v. 20, n. 5, p. 555-560, 2012.

HOFFMAN, B. D.; ANDERSEN, A. N. Responses of ants to disturbance in Australia, with particular reference to functional groups. Austral Ecology, v. 28, n. 4, p. 444-464, 2003.

HOFFMAN, B. D. Using ants for rangeland monitoring: Global patterns in the responses of ant communities to grazing. Ecological Indicators, v.10, n. 2, p. 105-111, 2010.

HOLEC, M.; FROUZ, J. Ant (Hymenoptera: Formicidae) communities in reclaimed and unreclaimed brown coal mining spoil dumps in the Czech Republic. Pedobiologia, v. 49, n. 4, p. 345-357, 2005.

HOLEC, M.; FROUZ, J.; POKORNÝ, R. The influence of different vegetation patches on the spatial distribution of nests and the epigeic activity of ants (Lasius niger) on a spoil dump after brown coal mining (Czech Republic). European Journal of Soil Biology, v. 42, n. 3, p. 142-165, 2006.

ILHA, C.; LUTINSKI, J. A.; PEREIRA D. V. M.; GARCIA, F. R. M. Riqueza de formigas (Hymenoptera: Formicidae) da Bacia da Sanga Caramuru, município de Chapecó-SC. Biotemas, v. 22, p. 95-105, 2009.
Instituto Brasileiro de Mineração (IBRAM). Ferro. In: Informações e Análises da Economia Mineral Brasileira. 6ed. Brasília, p. 14-15, 2011.

LEAL, I. R. FILGUEIRAS, B. K. C.; GOMES, J. P.; IANNUZZI, L.; ANDERSEN, A. N. Effects of habitat fragmentation on ant richness and functional composition in Brazilian Atlantic forest. Biodiversity Conservation, v. 21, n. 7, p. 1687-1701, 2012.

LEAL, L. C.; ANDERSEN, A. N.; LEAL, I. R. Anthropogenic disturbance reduces seed-dispersal services for myrmecochorous plants in the Brazilian Caatinga. Oecologia, v.174, n. 1, p. 173-181, 2014.

LOMOV, B.; KEITH, D. A.; HOCHULI, D. F. Linking ecological function to species composition in ecological restoration: Seed removal by ants in recreated woodland. Austral Ecology, v. 34, n. 7, p. 751-760, 2009.

MADEIRA, B. G.; ESPÍRITO-SANTO, M. M.; NETO, S. D. Â.; NUNES, Y. R.; AZOFEIFA, G. A. S.; FERNANDES, G. W.; QUESADA, M. Changes in tree and liana communities along a successional gradient in a tropical dry forest in south-eastern Brazil. Plant Ecology, v. 201, n. 1, p. 291-304, 2009.

MAJER, J. D.; DAY, J. E.; KABAY, E. D.; PERRIMAN, W. $S$. Recolonization by ants in bauxite mines rehabilitated by a number of different methods. Journal of Applied Ecology, v. 21, p. 355-375, 1984.

MAJER, J. D. Ant recolonisation of rehabilitated bauxite mines of Poços de Caldas, Brazil. Journal of Tropical Ecology, v. 8, n. 1, p. 97-108, 1992.

MAJER, J. D. Ant recolonization of rehabilitated bauxite mines at Trombetas, Pará, Brazil. Journal of Tropical Ecology, v. 12, n. 2, p. 257-273, 1996.

MAJER, J. D.; BRENNAN, K. E. C.; MOIR, M. L. Invertebrates and the Restoration of a Forest Ecosystem: 30 Years of Research following Bauxite Mining in Western Australia. Restoration Ecology, v. 15, n. s4, p. S104-S115, 2007.

MARINHO, C. G. S.; ZANETTI, R.; DELABIE, J. H. C.; SCHLINDWEIN, M. N.; RAMOS, L. D. S. Ant (Hymenoptera: Formicidae) diversity in eucalyptus (Myrtaceae) plantations and cerrado litter in Minas Gerais, Brazil. Neotropical Entomology, v. 31, n. 2, p. 187-195, 2002.

MCGEOCH, M. A. The selection, testing and application of terrestrial insects as bioindicators. Biological Reviews, v. 73, n. 1, p. 181-201, 1998. 
NEVES, F. S.; BRAGA, R. F.; ARAÚJO, L. S.; CAMPOS, R. I.; FAGUNDES, M. Differential effects of land use on ant and herbivore insect communities associated with Caryocar brasiliense (Caryocaraceae). Revista de Biologia Tropical, v. 60, n. 3, p. 1065-1073, 2012.

NIEMI, G. J., MCDONALD, M. E. Application of ecological indicators. Annual Review of Ecology, Evolution, and Systematics, v. 35, n. 1, p. 89-111, 2004.

PAIS, M. P.; VARANDA, E. M. Arthropod recolonization in the restoration of a semideciduous forest in southeastern Brazil. Neotropical entomology, v. 39, n. 2, p. 198-206, 2010.

PALACIO, E. E.; FERNÁNDEZ, F. Claves para las subfamilias y géneros. In: Fernández, F. (ed). Introduction a las hormigas de la Región Neotropical. Bogotá: Instituto Humboldt, Colômbia, p. 424.

PAOLUCCI, L. N.; SOLAR, R. R. C.; SOBRINHO, T. G.; SPERBER, C. F.; SCHOEREDER, J. H. How does smallscale fragmentation affect litter-dwelling ants? The role of isolation. Biodiversity Conservation, v. 21, n. 12, p. 3095-3105, 2012.

PARR, C. L.; ANDERSEN, A. N.; CHASTAGNOL, C.; DUFFAUD, C. Savanna fires increase rates and distance of seed dispersal by ants. Oecologia, v. 151, n. 1, p. 33-41, 2007.

PEREIRA, M. P. S.; QUEIROZ, J. M.; VALCARCEL, R.; MAYHÉ-NUNES, A. J. Fauna de formigas como ferramenta para monitoramento de área de mineração reabilitada na Ilha da Madeira, Itaguaí, RJ. Ciência Florestal, v. 17, n. 3, p. 197-204, 2007.

PEREIRA, M. F.; TRIGO, J. R. Ants have a negative rather than a positive effect on extrafloral nectaried Crotolaria pallida performance. Acta Oecologia, v. 51, p. 49-53, 2013.

PERFECTO, I.; VANDERMEER, J. Biodiversity conservation in tropical agroecosystems. Annals of the New York Academy of Sciences, v. 1134, n. 1, p. 173-200, 2008

PHILPOTT, S. M.; ARMEBRECHT, I. Biodiversity in tropical agroforests and the ecological role of ants and ant diversity in predatory function. Ecological Entomology, v. 31, n. 4, p. 369-377, 2006.

PHILPOTT, S. M.; PERFECTO, I.; ARMBRECHT, I.; PARR, C. Disturbance and habitat transformation. In.: Lach L, Parr C, Abbott K. (eds.). Ant Ecology. Oxford University Press. p. 137-156, 2010.
PIZO M. A.; OLIVEIRA P. S. Size and lipid content of nonmyrmecochorous diasporas: effects on the interaction with litter-foraging ants in the Atlantic rain forest of Brazil. Plant Ecology, v.157, p. 37-52, 2001.

QUEIROZ, A. C. M; RIBAS, C. R.; FRANÇA, F. M. Microhabitat characteristics that regulate ant richness patterns: the importance of leaf litter for epigaeic ants. Sociobiology, v. 60, n. 4, p. 367-373, 2013.

RAIMUNDO, R. L. G.; GUIMARAES, P. R.; ALMEIDA-NETO, N.; PIZO, M. A. The influence of fruit morphology and habitat structure on antseed interactions: A study with artificial fruits. Sociobiology, v. 44, n. 2, p. 261-270, 2004.

RAMOS, L. D.; FILHO, R. B. Z.; DELABIE, J.H. C.; LACAU, S.; SANTOS, M. F. S.; NASCIMENTO, I.C.; MARINHO, C. G. S. Ant communities (Hymenoptera: Formicidae) of the leaf-litter in cerrado "stricto sensu" areas in Minas Gerais, Brazil. Lundiana, v. 4, p. 95-102, 2003.

RIBAS, C. R.; SCHOEREDER, J. H.; PIC, M.; SOARES, S. M. Tree heterogeneity, resource availability and larger scale processes regulating arboreal ant species richness. Austral Ecology, v. 28, n. 3, p. 305-314, 2003.

RIBAS, C. R.; CAMPOS, R.; SCHMIDT, F. A.; SOLAR, R. R. C. Ants as Indicators in Brazil: A Review with Suggestions to Improve the Use of Ants in Environmental Monitoring Programs. Psyche: A Journal of Entomology, v. 2012, p. 1-23, 2012a.

RIBAS, C. R.; SCHMIDT, F. A.; SOLAR, R. R. C.; CAMPOS, R.; VALENTIM, C. L.; SCHOEREDER, J. $\mathrm{H}$. Ants as indicators of the success of rehabilitation efforts in deposits of Gold Mining Tailings.

Restoration Ecology, v. 20, n. 6, p. 712-720, 2012 b.

RIBAS, C. R.; SOLAR, R. C. C.; CAMPOS, R. B. F.; SCHMIDT, F. A.; VALENTIM, C. L.; SCHOEREDER, J. H. Can ants be used as indicators of environmental impacts caused by arsenic? Journal of Insect Conservation, v. 16, n. 3, p. 413-421, 2012c.

RIZALI, A.; CLOUGH, Y.; BUCHORI, D.; HOSANG, M. L. A.; BOS, M. M.; TSCHARNTKE, T. Longterm change of ant community structure in cacao agroforestry landscapes in Indonesia. Insect Conservation and Diversity, v.6, n. 3, p. 328-338, 2013.

RIZZINI, C. T. Tratado de fitogeografia do Brasil: aspectos ecológicos, sociológicos e florísticos. Âmbito Cultural, 1997.

SCHMIDT, F. A.; DIEHL, E. What is the effect of soil use on ant communities? Neotropical Entomology, v. 37, n. 4, p. 381-388, 2008. 
SCHMIDT, F. A.; RIBAS, C. R.; SCHOEREDER, J. H. How predictable is the response of ant assemblages to natural forest recovery? Implications for their use as bioindicators. Ecological Indicators, v. 84, p. 158-166, 2013.

SILVA, E. N; PERFECTO, I. Coexistence of aphid predators in Cacao plants: Does ant-aphid mutualism play a role? Sociobiology, v. 60, n. 3, p. 259-265, 2013.

SOUSA-SOUTO, L.; SCHOEREDER, J. H.; SCHAEFER, C. E. G. R. Leaf-cutting ants, seasonal burning and nutrient distribution in Cerrado vegetation. Austral Ecology, v. 32, n. 7, p. 758-765, 2007.

VASCONCELOS, H. L. Effects of forest disturbance on the structure of ground foraging ant communities in central Amazonia. Biodiversity and Conservation, v. 8, n. 3, p. 409-420, 1999.
VASCONCELOS, H. L.; VILHENA, J. M. S.; CALIBRI, G. J. A. Response of ants to selective logging of a central Amazonian forest. Journal of Applied Ecology, v.37, n. 3, p. 508-514, 2000

WALKER, L. R.; DEL MORAL, R. Primary Succession and Ecosystem Rehabilitation. Cambridge University Press, Cambridge, 2003.

WEISER, M. D.; KASPARI, M. Ecological mosphospace of New World ants. Ecological Entomology, v. 31, p. 131-142, 2006.

WILSON, E. O. Editorial: On the future of the conservation biology. Conservation Biology, v. 14, n. 1, p. 1-3, 2000 . 